\title{
Hiperparatiroidismo primario: presentación de un caso y revisión de la literatura
}

\author{
CAROLINA GARFIAS VON F. ${ }^{1}$, SOLEDAD VILLANUEVA T. ${ }^{1}$, FRANCISCA UGARTE P. ${ }^{2,3}$ \\ 1. Médico Pediatra, Unidad de Endocrinología, Hospital Dr. Exequiel González Cortés. \\ 2. Endocrinólogo Infantil, Unidad Endocrinología Hospital Dr. Exequiel González Cortés. \\ 3. Departamento de Pediatría, Universidad de Los Andes.
}

\begin{abstract}
Primary hyperparathyroidism: clinical case and literature review

Hypercalcemia is infrequent in pediatrics, causes include mutations of calcium sensing receptor (CaSRs), PT adenoma or hyperplasia, D or A hypervitaminosis, inborn errors of metabolism, parenteral nutrition, and others. Objective: To report a case of severe hypercalcemia in a adolescent due to primary hyperparathyroidism. Case: Fourteen years old adolescent girl with 2 weeks of weight loss, polyuria, malaise and emotional lability. Laboratory reveals hypercalcemia (16.6 mg/dl), hypophosphemia (2.2 mg/dl) and elevated PTH (450 $\mathrm{pg} / \mathrm{ml}$ ). Management of severe hypercalcemia at ICU was done. PT Scintigraphy study reveals increased uptake in the lower pole of right thyroid lobe. Right inferior parathyroidectomy was performed and biopsy revealed right lower parathyroid hyperplasia. Discusion: Primary hyperparathyroidism (HPT) is an uncommon condition in children. The main causes are parathyroid adenomas or hyperplasia, frequently one or two PT glands involved. HPT must be suspected in symptomatic hypercalcemia, nephrourinary symptoms in scholars and adolescents (polyuria and nephrolithiasis) and in newborn with pathologic fractures and costal rosary. The pathogenesis includes mutations in CaSRs, cyclin D1/PRAD 1 and MEN 1 genes.
\end{abstract}

(Key words: Hypercalcemia, hyperparathyroidism, primary hyperparathyroidism, calcium sensing receptor (CaSRs), child).

Rev Chil Pediatr 2011; 82 (4): 336-343

\section{RESUMEN}

La hipercalcemia es infrecuente en pediatría, existen diferentes causas que incluyen mutaciones del receptor sensible al calcio (CaSRs), adenoma o hiperplasia de PT, hipervitaminosis D o A, errores congénitos del metabolismo, nutrición parenteral total, etc. Objetivo: Comunicar un caso de hipercalcemia severa en una adolescente causado por un hiperparatiroidismo primario. Caso: Escolar de 14 años con cuadro de 2 semanas de baja de peso, poliuria, compromiso del estado general y labilidad emocional. Los exámenes revelaron hiper-

Trabajo recibido el 30 de junio de 2011, devuelto para corregir el 20 de julio de 2011, segunda versión el 28 de julio de 2011 , aceptado para publicación el 08 de agosto de 2011.

Correspondencia a:

Carolina Garfias von Furstenberg

Email:cpgarfias@gmail.com 
calcemia (16 mg/dl), hipofosfemia (2,2 mg/dl) y PTH elevada (450 pg/ml). Se hospitalizó para manejo de hipercalcemia severa, con diagnósticos de Hiperparatiroidismo primario. Se completó estudio con cintigrama de PT, encontrando hipercaptación en polo inferior de lóbulo tiroideo derecho. Se realizó una paratiroidectomia inferior derecha y la biopsia reveló hiperplasia de paratiroides inferior derecha. Discusión: El hiperparatiroidismo primario es una condición infrecuente en niños. Las principales causas son adenomas o hiperplasia de paratiroides, frecuentemente con 1 o dos glándulas PT comprometidas. Debe sospecharse en casos de hipercalcemia sintomática, escolares o adolescentes con síntomas nefrourinarios (poliuria importante y nefrolitiasis) y frente a un recién nacido grave, con fracturas patológicas o rosario costal. La etiopatogenia incluye mutaciones del gen CaSRs, Ciclin D1/PRAD 1 y el MEN 1.

(Palabras clave: Hipercalcemia, hiperparatiroidismo, hiperparatiroidismo primario, receptor sensible al calcio (CaSR), niños).

Rev Chil Pediatr 2011; 82 (4): 336-343

\section{Introducción}

En la edad pediátrica, la hipercalcemia es una alteración hidroelectrolítica poco frecuente a diferencia de lo observado en población adulta. La hipercalcemia se define como un calcio total mayor a $10,5 \mathrm{mg} / \mathrm{dl}$. En la tabla 1 se muestran los valores normales de calcio sérico según edad. ${ }^{1}$ Los síntomas y signos de hipercalcemia son variados y pueden presentarse con calcemias totales $>12 \mathrm{mg} / \mathrm{dl}$; las crisis hipercalcémicas se observan con calcemias $>15 \mathrm{mg} / \mathrm{dl} .{ }^{1,2}$

Las causas de hipercalcemia difieren entre neonatos, lactantes y niños mayores (tablas 2 y 3$)^{3}$. Las manifestaciones clínicas también difieren según la edad, siendo en neonatos y lactantes inespecíficas. En general, la hipercalcemia sintomática se puede manifestar con náuseas, vómitos, anorexia, compromiso de conciencia (desde irritabilidad, somnolencia hasta estupor y convulsiones); poliuria que puede llegar hasta la diabetes insípida nefro-

Tabla 1. Valores normales de calcio sérico, según edad

\begin{tabular}{|lc|}
\hline Edad (años) & Calcemia total (mg/dl) \\
\hline $0-1$ & $8,8-11,3$ \\
$1-5$ & $9,4-10,8$ \\
\hline $6-12$ & $9,4-10,3$ \\
\hline $12-20$ (mujeres) & $8,8-10$ \\
\hline $12-20$ (hombres) & $9,1-10,2$ \\
\hline
\end{tabular}

Modificado de Steven A y cols. ${ }^{3}$ génica, por estimulación de receptor sensible al calcio (CaSRs) en el túbulo colector renal, con la consiguiente disminución de la expresión de las aquaporinas 2 a nivel de la membrana apical; nefrocalcinosis, bradicardia y debilidad muscular proximal. El electrocardiograma puede mostrar bradicardia sinusal con acortamiento de QT. En neonatos podemos encontrar también deformidades esqueléticas severas, rosario costal y fracturas patológicas, que demuestran la alteración en la mineralización ósea y la intensa actividad osteoclástica ${ }^{2,3}$. La causa más frecuente en recién nacidos es la hiperplasia de glándulas paratiroides (PT) y la más grave es el hiperparatiroidismo neonatal severo, por mutación inactivante de los dos alelos que codifican el CaSRs ${ }^{3}$.

El objetivo del presente trabajo es dar a conocer el caso de una adolescente previamente sana, que presentó hipercalcemia sintomática causada por un hiperparatiroidismo primario.

\section{Caso clínico}

Paciente de 14 años 2 meses, de sexo femenino, sin antecedentes mórbidos personales de importancia. Padres y tres hermanas sanas, y abuelo paterno con Diabetes Mellitus tipo 2. Menarquia a los 12 años. Historia actual caracterizada por una semana de fiebre intermitente y disuria, diagnosticándose en forma ambulatoria infección urinaria febril que se trató con cefradoxilo. A pesar del tratamiento antibiótico persistió febril, agregándose dolor retroes- 
Tabla 2. Causas de hipercalcemia en neonatos y lactantes

Hipercalcemia en neonatos y lactantes
I. RNPT con lactancia materna o fórmula sin suplementación
de fosfato
II. Nutrición parenteral total
III. Hiperparatiroidismo
- Hiperplasia de PT (paratidoides) congénita
- Hipoparatiroidismo materno
IV. Mutaciones inactivantes del gen del CaSRs
- Hipercalcemia hipocalciurica familiar
- Hiperparatiroidismo neonatal severo
V. Condroplasia metafisiaria de Jansen
VI. Hipervitaminosis D
VII. Persitencia de PTHrP (péptidos relacionado a la PTH)
VIII. Síndrome de Williams / Hipercalcemia infantil idiopática
IX. Errores congénitos del metabolismo (deficiencia de lactasa,
intolerancia disacaridasas, síndrome de Bartter)

Modificado de Steven A y cols. ${ }^{3}$

ternal punzante, labilidad emocional, dolor abdominal intermitente tipo cólico, constipación (7 días), baja de peso (3 kilos en 3 semanas) y poliuria. Después de 21 días de evolución se hospitalizó con el diagnóstico de síndrome febril prolongado.

$\mathrm{Al}$ ingreso destacaba orina completa normal, hemograma con 14000 leucocitos (VN 4 000-10 000 GB), 62\% de segmentados. Hematocrito 39\% (VN 37-44\%), PCR: 43,2 mg/l (hasta $5 \mathrm{mg} / \mathrm{l}$ ), electrolitos plasmáticos normales, creatinina 0,62 mg/l (VN 0,4-1,0 mg/l), BUN 11,9 mg/dL (VN 7 a $20 \mathrm{mg} / \mathrm{dL}$ ), calcemia total 16,6 mg/dL (VN 8,5 a 10,2 mg/dL) y corregida 17,2 mg/dl, fosfemia 2,2 (VN 2,4 a 4,1 mg/dl), magnesio 1,2 mg/dL (VN 1,7 a $2,2 \mathrm{mg} / \mathrm{dL}$ ) y hemocultivos negativos. De los exámenes de imágenes destacaban radiografía de tórax y de cavidades paranasales normales, ecografía abdominal con hiperecogenicidad renal bilateral inespecífica, resto dentro de lí-
Tabla 3. Causas de hipercalcemia en mayores de 2 años y adolescentes

\begin{tabular}{|c|c|}
\hline Нiр & arcalcemia en niños mayores de 2 años y adol \\
\hline I. & Ingesta excesiva de calcio \\
\hline II. & Depleción de fosfato \\
\hline III. & Nutrición parenteral \\
\hline IV. & $\begin{array}{l}\text { Hiperparatiroidismo } \\
\text { - Primario Adquirido } \\
\text { - Adenoma } \\
\text { - Hiperplasia } \\
\text { - Carcinoma } \\
\text { - Genético } \\
\text { - Hipercalcemia Hipocalciurica Familiar } \\
\text { - MEN I y lla } \\
\text { - Hiperparatiroidismo terciario (autónomo) }\end{array}$ \\
\hline V. & $\begin{array}{l}\text { Hipervitaminosis D: exceso de ingesta, VIH y enfermeda- } \\
\text { des granulomatosas (sarcoidosis, tuberculosis, histoplas- } \\
\text { mosis, coccidiomycosis) }\end{array}$ \\
\hline VI. & Síndrome de Williams \\
\hline VII. & Hipercalcemia Idiopatica infantil \\
\hline VIII. & Condroplasia Metafisiaria de Jansen \\
\hline IX. & Inmovilización \\
\hline $\mathrm{x}$ & $\begin{array}{l}\text { Neoplasias (Hematológicas, tumores óseos primarios, } \\
\text { tumores metastáticos con osteolisis, tumores secretores } \\
\text { de PTHrP, prostaglandinas, citokinas y factores de creci- } \\
\text { miento) }\end{array}$ \\
\hline $\mathrm{XI}$. & Enfermedad hepática \\
\hline XII. & Hipertiroidismo \\
\hline XIII. & Insuficiencia suprarrenal \\
\hline XIV. & Feocromocitoma \\
\hline $\mathrm{XV}$. & $\begin{array}{l}\text { Drogas: tiazidas, litio, retinoides sistémicos, teofilina, } \\
\text { ácido acetilsalicilico }\end{array}$ \\
\hline $\mathrm{XVI}$. & Síndrome de leche alcali \\
\hline $\mathrm{XVII}$. & Acidosis tubular renal (ATR) \\
\hline
\end{tabular}

Modificado de Steven A. y cols ${ }^{3}$

mites normales. Se trasladó a la Unidad de Paciente Crítico (UPC) del Hospital Dr. Exequiel González Cortés, para estudio y manejo de su hipercalcemia severa.

Ingresó afebril, Glasgow 15, hemodinámicamente estable, con episodios de bradicardia sinusal asintomática. El manejo agudo de su hipercalcemia se realizó con hiperhidratación, corticoides endovenosos (hidrocortisona), aporte de fosfato monopotásico, con lo cual se produjo un descenso progresivo del calcio y regularización del resto de los electrolitos (tabla 4). 
Tabla 4. Caso clínico, exámenes de laboratorio

\begin{tabular}{|lccrrrrr|}
\hline $\begin{array}{l}\text { Días desde el comienzo } \\
\text { del estudio }\end{array}$ & $\mathbf{1}$ & $\mathbf{3}$ & $\mathbf{2 2}$ & $\mathbf{2 4}$ & $\mathbf{3 0}$ & $\mathbf{3 6}$ & $\mathbf{4 8}$ \\
\hline Calcio total $(\mathrm{mg} / \mathrm{dl})$ & 16,6 & 15,4 & 12,1 & 8,3 & 8,8 & 8,5 & 9,1 \\
\hline Fósforo $(\mathrm{mg} / \mathrm{dl})$ & 2,0 & 2,6 & 2,8 & 3,0 & 5,2 & 4,5 & 4,3 \\
\hline PTH $(\mathrm{pg} / \mathrm{ml})$ & & 450 & cirugía & 82,4 & 174,1 & 70,1 & \\
\hline Ca U/ Crea U* & & 0,17 & 0,79 & & & & 0,003 \\
\hline
\end{tabular}

Calciuria/ Creatininuria.

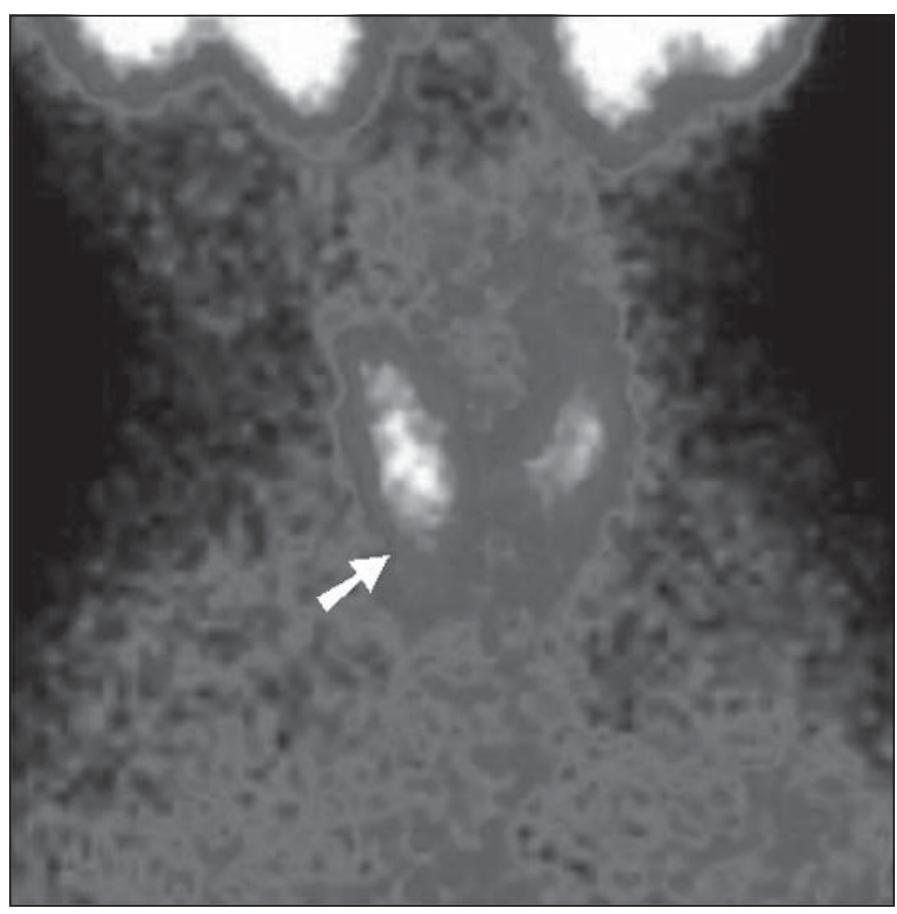

Figura 1. Cintigrama Tc99mMIBI de PT reveló leve concentración anormal del trazador en relación polo inferior de lóbulo tiroídeo derecho, sospechoso de adenoma paratiroídeo derecho.
La parathormona (PTH) fue de $>450 \mathrm{pg} /$ $\mathrm{ml}$ (VN 14-72), con calcemia de $15,4 \mathrm{mg} / \mathrm{dl} \mathrm{y}$ fosfemia de 2,6 mg/dl; calciuria/creatininuria 0,17. Dentro del estudio de segunda línea se solicitó ecografía cervical que resultó normal, se repitió la ecografía abdominal que mostró lesiones focales esplénicas múltiples hipoecogénicas de $<3 \mathrm{~mm}$; nefromegalia bilateral con buena diferenciación corticomedular. La tomografia axial computada (TAC) de abdomen y pelvis mostró múltiples lesiones hipodensas focales esplénicas de carácter indeterminado, por lo cual se sugirió descartar proceso linfo- proliferativo o de carácter infeccioso.

Se plantearon entonces los siguientes diagnósticos: a) Hipercalcemia en estudio: Hiperparatiroidismo ¿Primario?, Observación ¿̇neoplasia oculta?; b) Sd febril prolongado en estudio, de etiología ¿infecciosa? ¿neoplásica?.

Dentro del estudio realizado destacó la serología positiva IgM para Bartonella Henselae, confirmando una Enfermedad por Arañazo de Gato, que explicaba el síndrome febril prolongado y las lesiones esplénicas encontradas en la TAC, indicándose tratamiento con macrólidos.

Para completar el estudio de la hipercalce- 
mia con PTH elevada se realizó un cintigrama PT con Tc99mMIBI (Sestamibi-Tc99) que demostró leve concentración anormal del trazador en relación polo inferior de lóbulo tiroideo derecho, sospechoso de adenoma paratiroideo derecho. El cintigrama óseo reveló captación focal de radiofármaco en la calota (hiperostosis frontal interna) y resto del examen normal. Se completó el estudio para descartar Neoplasia Endocrina Múltiple (NEM) encontrándose Prolactina: 35,3 ng/mL (VN: 2 a $29 \mathrm{ng} / \mathrm{mL}$ ), TSH: 1,45 uU/ml (0,4-4,2 uU/mL), T4L: 0,91 ng/dl (VN 0,8-2,7 ng/dL), IGF-1: 348 ng/ml (VN: 359-639). Se difirió el estudio del eje suprarrenal por estar con tratamiento corticoidal.

Se realizó una paratiroidectomía inferior derecha a los 22 días de hospitalización. La evolución postquirúrgica fue sin incidentes, con normalización de electrolitos (Ca y P). Al sexto día postquirúrgico se dió el alta, en tratamiento con corticoides en dosis sustitución (12 $\mathrm{mg} / \mathrm{m}^{2} /$ día). La biopsia reveló una hiperplasia de paratiroides.

Acudió a control ambulatorio a los 4 días del alta en policlínico de endocrinología, encontrándose a la paciente en buenas condiciones generales, con IMC 17,65 (p24), talla 140 cm (p6) y asintomática. Al examen físico destacaba la tiroides normal y una cicatriz cervical en buenas condiciones, mamas Tanner IV, vello púbico Tanner III. Los exámenes de control como Ca, P, FA, PTH estaban dentro de rangos normales. La Densitometría Mineral Osea de columna volumétrica mostró z-score $-0,7$, columna cervical z-score - 1 y cuerpo total z-score -2,0. A los 15 días post quirúrgico se controló con un cintigrama paratiroideo que resultó negativo para adenoma/hiperplasia. Mantiene corticoides en dosis mínima de sustitución, con controles de cortisol matinal, en espera de probar suficiencia suprarrenal.

Los diagnósticos finales fueron: Hiperparatiroidismo Primario por Hiperplasia de Paratiroides Inferior Derecha, Osteopenia secundaria, Enfermedad por Arañazo de Gato tratada, Adolescente Eutrófica.

Se realizó una revisión sistemática de la literatura en las bases de datos de Pub Med, RIMA y Scielo, utilizando las palabras claves hipercalcemia, hiperparatiroidismo, hiperpara- tiroidismo primario, receptor sensible al calcio (CaSR) y niños.

\section{Discusión}

El hiperparatiroidismo es un estado de hipersecreción de paratohormona (PTH) por las glándulas paratiroides (PT). Se clasifica en primario, cuando existe excesiva secreción de PTH debido a una hiperplasia, adenoma o carcinoma de PT; secundario a hipocalcemia o deficiencia de Vitamina D y el llamado terciario, debido una función autónoma de las glándulas PT generalmente después de un hiperparatiroidismo secundario de larga evolución, como en la enfermedad renal crónica 5 .

La incidencia de hiperparatiroidismo primario (HPT $1^{\circ}$ ) en población pediátrica aún es desconocida, algunas han reportado incidencia de alrededor de 1 por cada 200000 a 300000 niños ${ }^{6}$. Otros autores han descrito que $5 \%$ del total de HPT $1^{\circ}$ se presentan en población pediátrica en igual proporción en hombres y mujeres ${ }^{6}$. En la población adulta el HPT es el tercer desorden endocrino más frecuente, con incidencia en los últimos 20 años entre 20-30 por 100000 personas al año ${ }^{8,9}$. Se ha observado una tendencia a la disminución en la incidencia en adultos los últimos años, las causas no están bien establecidas; se cree que el uso de terapia hormonal de reemplazo en mujeres post menopáusicas, cambios dietarios y la disminución de radiación ionizante de cabeza y cuello, podrían ser factores contribuyentes ${ }^{8,5}$.

Dentro de las causas de HPT $1^{\circ}$ están la hiperplasia, los adenomas y carcinomas de PT. Los adenomas son la principal causa en adultos (75-85\%), siendo lo más frecuente un adenoma único de una glándula PT superior (dos glándulas comprometidas 2-12\% y tres a cuatro glándulas < 1\%); 15\% de los pacientes presentan hiperplasia multiglandular, menos del 1\% carcinoma de PT, glándulas ectópicas se pueden observar en el $4-16 \%$ de los casos, siendo el mediastino, esófago y bajo el ángulo de la mandíbula los lugares más comunes ${ }^{5}$. En las distintas cohortes pediátricas se ha descrito mayor frecuencia de compromiso de las 4 glándulas que adenomas únicos. ${ }^{6,7}$ 
La etiopatiogenia de esta enfermedad es bien conocida. Se han descrito mutaciones del CaSRs, este receptor es codificado por un gen ubicado en el cromosoma 11, se ubica en la superficie de las células PT y su función es preservar la homeostasis del calcio censando los valores de éste en el plasma y regulando la secreción de PTH. La mutación de éste gen genera una alteración en el set point del receptor, que se vuelve "insensible" a las variaciones de la calcemia, generando así un aumento de la secreción de PTH con hiperparatiroidismo y aumento de la masa de tejido PT. Este tipo de mutación se observa en hiperplasia, adenomas e hiperparatiroidismo neonatal severo $(\text { HPTNS })^{10}$. Otra causa descrita es la mutación del gen Ciclin D1/Prad 1 un oncogén ubicado en el cromosoma 11q13 que codifica la ciclin D1, proteína que actúa en el ciclo celular; se genera una sobreproducción de esta proteína con la consiguiente hiperplasia o adenoma de paratiroides; esta mutación es responsable del 20-40\% de adenomas esporádicos (no familiares $)^{11}$. Otra mutación descrita es la autosómica dominante inactivante del gen MEN 1, gen supresor de tumores ubicado en el cromosoma 11, esta mutación produce adenoma de PT, neoplasia enteropancreática, hipofisiaria y suprarrenal, entidad conocida como Neoplasia Endocrina Múltiple tipo 1 (MEN 1), responsable del 20\% de adenomas esporádicos de PT y $5 \%$ de casos familiares. Otro gen involucrado es el protooncogen RET que codifica para una proteína tirosina kinasa, responsable del MEN 2A (Neoplasia Endocrina Múltiple tipo 2A), la cual se caracteriza por neoplasia medular de tiroides (95\% casos), feocromocitoma (5\% casos) e hiperparatiroidismo en $20 \%$ pacientes. El HPT es usualmente leve y asintomático en los pacientes con MEN 2A y secundario a hiperplasia multiglandular ${ }^{12}$.

El hiperparatiroidismo neonatal severo (HPTNS) es una entidad poco frecuente y grave que se produce por mutación autosómica recesiva del gen que codifica el CaSR, produciendo hiperplasia de paratiroides (generalmente las 4 comprometidas), con hipercalcemia grave, importante compromiso óseo, deformaciones como rosario costal, fracturas patológicas, lo que demuestra gran altera- ción de la mineralización ósea y actividad osteoclástica intensa ${ }^{3,4}$.

A diferencia del adulto, en que la hipercalcemia es generalmente asintomática y constituye un hallazgo de laboratorio, los pacientes pediátricos son sintomáticos en más de un 70\% de las ocasiones. Los síntomas y signos son secundarios a la hipercalcemia crónica, como astenia, compromiso del estado general, pérdida de peso (39\%); manifestaciones renales (41\%) como hipercalciuria, nefrolitiasis y síndrome de diabetes insípida nefrogénica; manifestaciones musculoesqueléticos (16\%) como atrofia muscular, debilidad, hiperreflexia y osteoporosis; manifestaciones gastrointestinales (25\%) como náuseas, vómitos y dolor abdominal; y síntomas neuropsiquiátricos (8\%) como irritabilidad, demencia y confusión. La hipercalcemia asintomática se describe sólo en un $18 \%$ de los niños ${ }^{5,6}$. En nuestra paciente los síntomas que más destacaron fueron baja de peso, astenia, compromiso del estado general, poliuria, dolor abdominal, constipación e irritabilidad.

Dentro de los exámenes de laboratorio en población pediátrica, se describe hipercalcemia con valores entre 11,2 a 18 mg/dl (en el HPNS se han descrito calcemias totales de hasta 25 $\mathrm{mg} / \mathrm{dl}$ ), la paratohormona (PTH) generalmente se encuentra elevada o dentro de rangos normales lo cual se considera anormal en el contexto de una hipercalcemia, las elevaciones pueden ser leves (80-90 pg/ml) o alcanzar niveles hasta 1500 pg/ml (VN 14-72 pg/ml), nuestra paciente debutó con PTH en rango intermedio 450 pg/ $\mathrm{ml}$. La fosfemia y la Vitamina D se encuentran normal en la mayor parte de los casos. Hipercalciuria se observa en más del 70\% de los niños con HPT $1^{\circ}$ con relación calciuria/creatininuria alterada para la edad.

El estudio con imágenes es fundamental para el diagnóstico y tratamiento de esta enfermedad, en población infantil se ha descrito una sensiblidad de alrededor de $84 \%$ en la ecocografia doppler de cuello para diagnosticar aumentos de tamaño de glándulas PT, al combinar el cintigrama con metoxiisobutylinitrilo (MIBI)/Tc ${ }^{99}$ con el Eco Doppler la sensibilidad aumenta hasta $90 \%{ }^{5,13}$. La DMO puede mostrar osteopenia u osteoporosis. 
Dentro de los diagnósticos diferenciales están todas las causas descritas anteriormente de hipercalcemia (tablas 2 y 3). Un diagnóstico importante de mencionar en un paciente con hipercalcemia y elevación moderada de PTH es la Hipercalcemia Hipocalciúrica familiar, condición benigna producida por una mutación autosómica dominante del CaSR pero con penetrancia incompleta a nivel renal y de PT. En esta, se produce hipersecreción de PTH pero con hipocalciuria, por aumento de la reabsorción tubular de calcio. Generalmente son pacientes asintomáticos y el diagnóstico se realiza muchas veces después de una paratiroidectomía fallida o cuando existen antecedentes familiares $^{9}$.

Con respecto al tratamiento en población adulta existen consensos y criterios claros de tratamiento médico y quirúrgico ${ }^{14,15}$. Cuando el HPT es sintomático el tratamiento es siempre quirúrgico, pero frecuentemente se realiza tratamiento conservador. En población pediátrica no existen consensos sobre su manejo debido a la baja incidencia, pero los expertos recomiendan ${ }^{16}$ siempre un manejo quirúrgico con exploración cervical bilateral, debido al gran número de pacientes en éste grupo etáreo con compromiso poliglandular; la cirugía mínimamente invasiva aún es discutible en éste grupo. La cirugía debe ser realizada por cirujanos con experiencia en cabeza y cuello ${ }^{16}$. El ideal es medir durante la cirugía PTHi (PTH intacta) ya que con una disminución del 50\% bajo el valor basal del paciente, después de 5-10 minutos de exéresis de las glándulas, se considera una paratiroidectomia satisfactoria ${ }^{9}$.

Con respecto al tratamiento médico es importante mencionar que en casos de hipercalcemia grave y sintomática, como ocurrió en nuestra paciente, se debe realizar un manejo agudo de la hipercalcemia en unidades de pacientes crítico. El manejo agudo de la hipercalcemia escapa al objetivo de nuestra revisión y se puede encontrar en referencia $17^{17}$.

Interesante es comentar la experiencia del Johns Hopkins Hospital ${ }^{7}$, entre los años 1984 y 2001, donde se reportaron 16 pacientes entre 10,5 y 20 años con HPT 1, de los cuales eran 7 mujeres y 9 hombres; dentro de los exámenes destacaban calcemias entre 10,6-14 mg/dl y la PTH entre 31-2 130 pg/ml, sólo en $71 \%$ de los pacientes se realizó cintigrama con Sestamibi Tc ${ }^{99}$; del total 11 pacientes (69\%) tenían adenoma de 1 paratiroides, 1 paciente (6\%) con adenoma de 2 PT y 4 (25\%) pacientes con presentó hiperplasia de 4 PT; en todos los pacientes se realizó manejo quirúrgico y el diagnóstico definitivo se confirmó con la anatomía patológica. Los franceses reportaron su experiencia entre los años 1984 y $2004^{6}$ con 55 casos de HPT $1^{\circ}$, de los cuales 11 (20\%) eran neonatos que presentaban calcemias entre 11 y 25 mg/dl y PTH entre 56 y 2214 pg/ml; el estudio genético del CaSR se realizó en tres pacientes encontrándose alterado sólo en 2, con mutaciones del exón 3 (mutación Y161c, correspondiendo a un fenotipo de HPT $1^{\circ}$ neonatal moderado) y del exón 7 (C582Y, que corresponde a un fenotipo más severo); dentro de los síntomas encontrados se describen hipotonía, dificultad en la alimentación, distress respiratorio, deformaciones torácicas, muchos de estos síntomas son inespecíficos y se observan en otras patologías del recién nacido. De los 55 pacientes con $\mathrm{HPT} 1^{\circ} 44$ pacientes (80\%) eran niños, 26 mujeres y 18 hombres, con edad que fluctuaba entre 6 y 18 años, con una máxima incidencia entre los 13 y 16 años; los síntomas predominantes (41\%) fueron nefrourológicos (nefrolitiasis, poliuria, hematuria, diabetes insípida nefrogénica), síntomas generales (39\%) como astenia y pérdida de peso, $16 \%$ de los pacientes presentaron síntomas musculoesqueléticos y 8\% neuropsiquiátricos (depresión, irritabilidad). La calcemia al diagnóstico varió entre 11 a 18 mg/dl y la PTH entre 24 a 1500 $\mathrm{pg} / \mathrm{ml}$; en dos pacientes se diagnóstico MEN 1, 20 pacientes presentaron adenomas de PT, 11 hiperplasia y 3 tejido PT ectópico; se realizó eco doppler en el $100 \%$ de los pacientes y cintigrama de PT en 33\%; el manejo fue quirúrgico en el total de los casos, siendo no exitoso en 5 casos, en los cuales hubo que reintervenir; se realizó PTHi en 6 pacientes y 40\% de los pacientes se presentaron hipocalcemia sintomática post operatoria.

Podemos concluir, que a diferencia de la población adulta en pediatría el hiperparatiroidismo primario es una patología poco frecuente, sintomática y grave en la mayor parte de 
los casos; que las causas más frecuentes son hiperplasia o adenoma de paratiroides, que debe sospecharse en casos de hipercalcemia sintomática, en escolares o adolescentes con síntomas nefrourinarios (poliuria importante y nefrolitiasis) y frente a un recién nacido grave, con fracturas patológicas o rosario costal. Siempre se debe tener presente el estudio de otros ejes por posible neoplasia endocrina múltiple (NEM).

\section{Referencias}

1.- Moe S: Disorders involving calcium, phosphorus and magnesium. Prim Care 2008, 35 (2): 215.

2.- Aranguiz C, Trujillo O, Reyes ML: Emergencias Endocrinas en Pediatria. Rev Med Chile 2005, 133: 1371-80.

3.- Steven A, Lietman A, Emily L, Germaine L, Michel A: Hipercalcemia in children and adolescents. Curr Opin Pediatr 2010, 22: 508-15.

4.- Obermannova B, Banghova K, Sumnik Z: Unusually severe phenotype of neonatal primary hyperparathyroidism due to a heterozygous inactivating mutations of the CaSRs gene. Eur J Pediatr 2009; 168: 569-73.

5.- Ahmad R, Hammond J: Primary, secondary and tiertiary hyperparathyroidism. Otolaryngol Clin N Am 2004; 37: 701-13.

6.- Mallet E: Working Group On Calcium Metabolism. Primary Hyperparathyroidism in neonates and childhood. Horm Res 2008; 69: 180-18.

7.- Hsu E, Levine M: Primary Hyperparathyroidism in children and adolescents: The Johns Hopkins Children`s Center. J Bone Miner Res 2002; 17: 44-50.

8.- Wermers $R$, Khosla S, Atkinson E, et al: Incidence of primary Hyperparathyroidism in Rochester, Minnesota 1993-2001: An update on the changing epidemiology of disease. J Bone Miner Res 2006; 21 (1): 171-7.

9.- Fraser W: Hyperparathyroidism. Lancet 2009; 374: 145-58.

10.- Brown E: The Pathophysiology of primary hyperparathyroidism. J Bone Miner Res 2002; 17: 24-9.

11.- Andrew A, Shattuck T, Sanjay M, et al: Molecular pathogenesis of primary hyperparathyroidism. J Bone Miner Res 2002; 17: 30-6.

12.- Malone J, Srivasta A, Khardori R: Hyperparathyroidism and multiple endocrine neoplasia. Otolaryngol Clin $\mathrm{N}$ Am 2004; 37: 715-36.

13.- Stephen J, Marx M: Hyperparathyroid and Hypoparathyroid Disorders. N Engl J Med 2000; 343: 1863-75.

14.- Villabona C: Nuevo consenso de actitud ante el hiperparatiroidismo primario. Endocrinol Nutr 2009; 56 (6): 281-6.

15.- Bilezikian JP, Khan AA, Potts JT: Guidelines for the Management of Asymptomatic Primary Hyperparathyroidism: Summary Statement from the Third International Workshop. J Clin Endocrinol Metab 2009; 4 (2): 335-9.

16.- Cupisti K, Raffel A, Dotzenrath C, Krausch M, Roher $H D$, Sculte KM: Primary hiperparatiroidismo in the young age group: particularities of diagnostic and therapeutic schemes. W J Surg 2004; 28: 1153-6.

17.- Kearney T, Dang C: Diabetic and endocrine emergencies. Post Med J 2007; 83 (976): 79-86. 